\title{
Roots and Root Spaces of Compact Banach-Lie Algebras
}

\author{
A. J. CALDERÓN MARTÍN AND M. FORERO PIULESTÁN
}

\begin{abstract}
We study the main properties of roots and root spaces associated to a Cartan subalgebra of a compact Banach-Lie algebra. As a consequence, we describe the topologically simple compact Banach-Lie algebras having a Cartan decomposition relative to some Cartan subalgebra.
\end{abstract}

\section{INTRODUCTION}

A Banach Lie algebra $L$ is a complex Lie algebra such that its underlying vector space is a Banach space and satisfies $\|[x, y]\| \leq 2\|x\| \cdot\|y\|$ for every $x, y \in L$. We shall say that $L$ is compact if the linear operators

$$
\operatorname{ad}(x): L \longrightarrow L, \quad \operatorname{ad}(x)(y)=[x, y],
$$

are compact for any $x \in L$ (see [2, Chapter IV] for a general reference on compact Banach algebras). This paper is a study of such algebras with emphasis, of course, on the infinite dimensional ones.

The paper is organized as follows. In the second section we study the roots and root spaces associated to a Cartan subalgebra of a compact Banach-Lie algebra, our main results in this section are that the root spaces associated to nonzero roots are one-dimensional and that the only integral multiplies $k \alpha$ of a nonzero root $\alpha$ which are roots are 0 and $\pm \alpha$. In the third section we study the direct limit of a direct system of Banach-Lie algebras, we prove the existence of the direct limit (unique up to isometric isomorphism), and we

2000 Mathematics Subject Classification. Primary 17B65, 46H70; Secondary $17 \mathrm{~B} 05$.

Supported by the PCI of the Spanish Junta de Andalucía 'Estudio analíticoalgebraico de sistemas triples y de pares en diferentes clases de estructuras no asociativas', by the PAI of the Spanish Junta de Andalucía, project number FQM-298, and by the Spanish DGICYT, project number PB97-1497. 
study some of its properties. Finally, in the fourth section we obtain a description of infinite dimensional topologically simple compact Banach-Lie algebras having a Cartan decomposition $L$, by approximating $L$ as the direct limit of a suitable family of simple finite dimensional Banach-Lie subalgebras. It is shown that this class can be realized as topologically simple Lie subalgebras of certain topologically simple associative Banach algebras. The results turn out to be natural extensions of the finite dimensional theory and the $L^{*}$-algebras theory of Schue (see $[7,8]$ ).

\section{Roots and Cartan Decompositions}

In order to make sense, the definition of Cartan subalgebra given below requires a definition of semisimplicity for infinite dimensional Banach-Lie algebras. Following [6], a Banach-Lie algebra is said to be semisimple if it has no non trivial abelian closed ideals. We define the annihilator of a Banach-Lie algebra $L$ as the closed ideal given by $\operatorname{Ann}(L)=\{x \in L:[x, y]=0$ for all $y \in L\}$. It is clear that any semisimple Banach-Lie algebra $L$ has zero annihilator, therefore $L$ is also semisimple in the sense given in [7] for $L^{*}$-algebras. We shall say that a Banach-Lie algebra $L$ is topologically simple if the product is nonzero and has no nonzero proper closed ideals. In the remainder of this section $L$ will denote a semisimple Banach-Lie algebra.

A Cartan subalgebra $H$ of $L$ is defined as a maximal abelian subalgebra; let us note that a Cartan subalgebra is necessarily closed. A root of $L$ relative to $H$ is a mapping $\alpha: H \longrightarrow \mathbb{C}$ such that there exists $v_{\alpha} \in L, v_{\alpha} \neq 0$ satisfying $\left[h, v_{\alpha}\right]=\alpha(h) v_{\alpha}$ for any $h \in H$. The root space associated to $\alpha$ is the subspace $L_{\alpha}=\left\{v_{\alpha} \in L:\left[h, v_{\alpha}\right]=\right.$ $\alpha(h) v_{\alpha}$ for any $\left.h \in H\right\}$. Given a set $S$ of nonzero roots of $L$, we shall denote by $S p_{\mathbb{Z}} S$ the set of mappings

$$
S p_{\mathbb{Z}} S=\left\{\sum_{i=1}^{n} p_{i} \alpha_{i}: p_{i} \in \mathbb{Z} \text { and } \alpha_{i} \in S\right\} .
$$

We shall say that $L$ has a Cartan decomposition relative to $H$ if

$$
L=H \oplus \overline{\bigoplus_{\alpha \in \Lambda} L_{\alpha}}
$$

where $\Lambda$ is the set of all nonzero roots of $L$ relative to $H$, and for any finite set $S \subset \Lambda$ we have that $S p_{\mathbb{Z}} S \cap \Lambda$ is also finite. Suppose that $L$ has a Cartan decomposition $L=H \oplus \overline{\bigoplus_{\alpha \in \Lambda} L_{\alpha}}$. We then 
recover the definition of Cartan subalgebra for finite dimensional Lie algebras, and for Lie algebras of arbitrary dimension given by Billing and Pianzola in [1]. Indeed, $H$ is nilpotent of length two, and it is easy to prove that $H$ is its own normalizer, $N(H)$, taking into account that given $x \in N(H)$ we have for any $h \in H$ that $[h, x] \in H \cap \overline{\bigoplus_{\alpha \in \Lambda} L_{\alpha}}=0$. Hence, by the maximal character as abelian subalgebra of $H, x \in H$. Let us observe finally that in case that $L$ has an involution our definition of Cartan subalgebra coincides with the one given by Schue for $L^{*}$-algebras in [7], and the one given by De la Harpe for $c$-involutive Lie algebras in $[6$, definition 4 on page 32], by adding to $H$ the condition of being also selfadjoint.

It is easy to prove that the roots are continuous and linear mappings, the root spaces are closed, the root space associated to the zero root is the Cartan subalgebra and, by the Jacobi identity, that if $\alpha+\beta$ is a root then $\left[L_{\alpha}, L_{\beta}\right] \subseteq L_{\alpha+\beta}$ and if $\alpha+\beta$ is not a root then $\left[L_{\alpha}, L_{\beta}\right]=0$.

Lemma 1. Let $\alpha$ and $\beta$ be two nonzero roots of $L$ relative to $H$. Then we have:

(1) There exists $n \in \mathbb{Z}$ such that $n \alpha$ is a root and $m \alpha,-m \alpha$ are not roots for any $m \in \mathbb{N}$ such that $m>|n|$.

(2) There exists $p \in \mathbb{Z}$ such that $\beta+p \alpha$ is a root and $\beta+q \alpha$, $\beta-q \alpha$ are not roots for all $q \in \mathbb{N}$ such that $q>|p|$.

Proof. For a root $\gamma$ of $L$ relative to $H$, we have $\|\gamma\| \leq 2$. Indeed, $|\gamma(h)| \leq\|h\|$ for any $h \in H$ and thus $\|\gamma\|=\sup _{h \in H}|\gamma(h)| /\|h\| \leq 2$.

(1) If $k \alpha$ is a root, as $\|k \alpha\| \leq 2$ then $|k| \leq \frac{2}{\|\alpha\|}$ and the result follows easily.

(2) If $\beta+k \alpha$ is a root then $2 \geq\|\beta+k \alpha\| \geq|k| \cdot\|\alpha\|-\|\beta\|$ and therefore (2) is clear.

Lemma 2. Let L be a semisimple compact Banach-Lie algebra, let $H$ be a Cartan subalgebra of $L$, and let $\alpha$ be a nonzero root of $L$ relative to $H$. Then the following assertions hold:

(1) The dimension of the root space $L_{\alpha}$ is finite;

(2) $-\alpha$ is a root;

(3) $\left[L_{\alpha}, L_{-\alpha}\right] \neq 0$.

Proof. (1) Fix $0 \neq h_{0} \in H$ and $0 \neq v_{\alpha} \in L_{\alpha}$, as $\operatorname{ad}\left(h_{0}\right)\left(v_{\alpha}\right)=$ $\alpha\left(h_{0}\right) v_{\alpha}$. Then $\alpha\left(h_{0}\right)$ is an eigenvalue of $a d\left(h_{0}\right)$ and $v_{\alpha} \in L\left(\alpha\left(h_{0}\right)\right)$, where $L\left(\alpha\left(h_{0}\right)\right)$ denotes the eigenspace associated to $\alpha\left(h_{0}\right)$. Hence 
$L_{\alpha} \subseteq L\left(\alpha\left(h_{0}\right)\right)$. As the operator $\operatorname{ad}\left(h_{0}\right)$ is compact, $\operatorname{dim} L\left(\alpha\left(h_{0}\right)\right)$ is finite and so is $\operatorname{dim} L_{\alpha}$.

(2) Let us suppose that $-\alpha$ is not a root. Fix $0 \neq h_{0} \in H$ such that $\alpha\left(h_{0}\right) \neq 0$, and let us consider $L^{\prime}:=\mathbb{C} h_{0} \oplus \mathbb{C} v_{\alpha} \oplus \bigoplus_{j=2}^{\infty} L_{-j \alpha}$, where $0 \neq v_{\alpha} \in L_{\alpha}$. It is easy to check that $L^{\prime}$ is a finite dimensional semisimple Lie algebra, $\mathbb{C} h_{0}$ is a Cartan subalgebra of $L^{\prime}$ and that $L^{\prime}$ has a Cartan decomposition relative to $\mathbb{C} h_{0}$, given by $L^{\prime}=\mathbb{C} h_{0} \oplus$ $L_{\alpha^{\prime}}^{\prime} \oplus \bigoplus_{j=2}^{\infty} L_{-j \alpha^{\prime}}^{\prime}$, where $\alpha^{\prime}=\left.\alpha\right|_{\mathbb{C} h_{0}}$ and $-j \alpha^{\prime}=-\left.j \alpha\right|_{\mathbb{C} h_{0}}$ are roots of $L^{\prime}$ relative to $\mathbb{C} h_{0}$, being then $L_{\alpha^{\prime}}^{\prime}=\mathbb{C} v_{\alpha}$ and $L_{-j \alpha^{\prime}}^{\prime}=L_{-j \alpha}$, $j \geq 2$. It is well known from the theory of finite dimensional split semisimple Lie algebras that if $\alpha^{\prime}$ is a root of $L^{\prime}$, then $-\alpha^{\prime}$ is also. We have therefore a contradiction.

The proof of (3) is similar by considering now $L^{\prime}:=\mathbb{C} h_{0} \oplus L_{\alpha} \oplus$ $\bigoplus_{j=1}^{\infty} L_{-j \alpha}$

Lemma 3. Let $L$ be a semisimple compact Banach-Lie algebra, let $H$ be a Cartan subalgebra of $L$, and let $\alpha, \beta$ be two nonzero roots of $L$ relative to $H$. Then we have:

(1) If $\beta+\alpha$ and $\beta-\alpha$ are not roots then $\beta\left(h_{\alpha}\right)=0$ for any $h_{\alpha} \in\left[L_{\alpha}, L_{-\alpha}\right]$;

(2) $\beta\left(h_{\alpha}\right)=r \alpha\left(h_{\alpha}\right)$ with $r \in \mathbb{Q}$ and $h_{\alpha}$ as above.

Proof. We obtain (1) as an easy consequence of the Jacobi identity and the identities $\left[L_{\alpha}, L_{\beta}\right]=\left[L_{\alpha}, L_{-\beta}\right]=0$.

(2) Let us fix $h_{\alpha}=\left[v_{\alpha}, v_{-\alpha}\right]$ with $v_{\alpha} \in L_{\alpha}$ and $v_{-\alpha} \in L_{-\alpha}$. Let us consider $V=\mathcal{L}\left(L_{\beta+j \alpha}: j \in \mathbb{Z}\right)$, the linear space generated by $\left\{L_{\beta+j \alpha}: j \in \mathbb{Z}\right\}$. By Lemmas 1-(2) and 2-(1), it is clear that $V$ is a finite dimensional vector space invariant for $a d\left(v_{\alpha}\right), a d\left(v_{-\alpha}\right)$ and $\operatorname{ad}\left(h_{\alpha}\right)=\operatorname{ad}\left(v_{\alpha}\right) a d\left(v_{-\alpha}\right)-a d\left(v_{-\alpha}\right) a d\left(v_{\alpha}\right)$. The fact that the trace of $a d\left(h_{\alpha}\right)$ on $V$ is 0 gives us the equation

$$
m \beta\left(h_{\alpha}\right)+k \alpha\left(h_{\alpha}\right)=0
$$

with $m \neq 0$ and $m, k \in \mathbb{Z}$. Hence $\beta\left(h_{\alpha}\right)=\frac{k}{m} \alpha\left(h_{\alpha}\right)$.

Corollary 1. Let $L$ be a semisimple compact Banach-Lie algebra having a Cartan decomposition relative to a Cartan subalgebra $H$, and let $\alpha$ be a nonzero root. Then $\alpha\left(h_{\alpha}\right) \neq 0$ for each nonzero $h_{\alpha} \in\left[L_{\alpha}, L_{-\alpha}\right]$. 
Proof. Let us suppose that $\alpha\left(h_{\alpha}\right)=0$. By Lemma 3-(2), $\beta\left(h_{\alpha}\right)=0$ for all roots $\beta$ and hence $\left[h_{\alpha}, L\right]=0$. Therefore $h_{\alpha} \in \operatorname{Ann}(L)$ and thus $h_{\alpha}=0$, a contradiction.

Theorem 1. Let $L$ be a semisimple compact Banach-Lie algebra having a Cartan decomposition relative to a Cartan subalgebra $H$, and let $\alpha$ be a nonzero root. Then $L_{\alpha}$ is one-dimensional and the only integral multiplies $k \alpha$ which are roots are 0 and $\pm \alpha$.

Proof. By Lemma 2-(3) we can fix $0 \neq v_{\alpha} \in L_{\alpha}$ and $0 \neq v_{-\alpha} \in L_{-\alpha}$ such that $0 \neq h_{\alpha}=\left[v_{\alpha}, v_{-\alpha}\right]$. Let us consider

$$
L^{\prime}=\mathbb{C}\left[v_{\alpha}, L_{-\alpha}\right] \oplus \mathbb{C} v_{\alpha} \oplus \bigoplus_{j=1}^{\infty} L_{-j \alpha}
$$

From Lemmas 1 and 2 it is easy to check that $L^{\prime}$ is a finite dimensional semisimple Lie algebra invariant for $a d\left(v_{\alpha}\right), \operatorname{ad}\left(v_{-\alpha}\right)$, and

$$
\operatorname{ad}\left(h_{\alpha}\right)=\left[\operatorname{ad}\left(v_{\alpha}\right), \operatorname{ad}\left(v_{-\alpha}\right)\right] .
$$

Since the trace of $a d\left(h_{\alpha}\right)$ on $L^{\prime}$ is 0 and since $\left[h_{\alpha}, v_{\alpha}\right]=\alpha\left(h_{\alpha}\right) v_{\alpha}$,

$$
\left[h_{\alpha}, v_{-j \alpha}\right]=-j \alpha\left(h_{\alpha}\right) v_{-j \alpha},
$$

and $\left[h_{\alpha}, h^{\prime}\right]=0$ for any $h^{\prime} \in \mathbb{C}\left[v_{\alpha}, L_{-\alpha}\right]$, we have

$$
\alpha\left(h_{\alpha}\right)\left(1+\sum(-j) d_{j}\right)=0
$$

where $d_{j}$ is the dimension of $L_{-j \alpha}$. By Corollary $1, \alpha\left(h_{\alpha}\right) \neq 0$, hence

$$
1+\sum(-j) d_{j}=0
$$

Thus $-2 \alpha,-3 \alpha, \cdots$ are not roots and $d_{-1}=1$. Since we can replace $\alpha$ by $-\alpha$ in the argument, we have both conclusions of the theorem.

The next corollary is now immediate

Corollary 2. Under the hypothesis of Theorem 1, if $\alpha, \beta$ and $\alpha+\beta$ are nonzero roots of $L$ relative to $H$ then $\left[L_{\alpha}, L_{\beta}\right]=L_{\alpha+\beta}$.

\section{Direct Limits of Banach-Lie Algebras}

Let $(I, \leq)$ be a directed set and let $\left\{L_{i}\right\}_{i \in I}$ be a family of BanachLie algebras such that for any $i, j \in I$ with $i \leq j$ there exists an isometric monomorphism $e_{j i}: L_{i} \longrightarrow L_{j}$ such that $e_{j i} \circ e_{i k}=e_{j k}$ and $e_{i i}=I d$ for all $i, j, k$ with $k \leq i \leq j$. Then we shall say that $S=\left(\left\{L_{i}\right\}_{i \in I},\left\{e_{j i}\right\}_{i \leq j}\right)$ is a direct system of Banach-Lie algebras. 
Given $S$ we define a direct limit, $\lim S$, as a couple $\left(L,\left\{e_{i}\right\}_{i \in I}\right)$ where $L$ is a Banach-Lie algebra, $e_{i}: L_{i} \longrightarrow L$ is an isometric monomorphism that satisfies $e_{i}=e_{j} \circ e_{j i}$ and $\left(L,\left\{e_{i}\right\}_{i \in I}\right)$ is universal for this property in the sense that if $\left(L^{\prime},\left\{t_{i}\right\}_{i \in I}\right)$ is another such couple, then there exists a unique isometric monomorphism $\theta$ from $L$ to $L^{\prime}$ such that $t_{i}=\theta \circ e_{i}$ for all $i \in I$. It is clear that if a direct limit exists, then it is unique up to isometric isomorphism. We define the concepts of direct system and direct limit for associative Banach algebras in a similar way.

If $A$ is an associative Banach algebra then $A^{-}$will denote the Banach-Lie algebra whose underlying vector space and norm agree with that of $A$ and whose product is given by $[x, y]=x y-y x$.

As in [3], we can prove that any direct system of Banach-Lie algebras $S$ has a direct limit, and state the following results.

Theorem 2. Let $S=\left(\left\{A_{i}\right\}_{i \in I},\left\{e_{j i}\right\}_{i \leq j}\right)$ be a direct system of associative Banach algebras. Then $S^{-}=\left(\left\{A_{i}^{-}\right\}_{i \in I},\left\{e_{j i}\right\}_{i \leq j}\right)$ is a direct system of Banach-Lie algebras and $\lim _{\rightarrow} S^{-}=\left(\lim _{\rightarrow} S\right)^{-}$.

Theorem 3. Let $S=\left(\left\{A_{i}\right\}_{i \in I},\left\{e_{j i}\right\}_{i \leq j}\right)$ be a direct system of associative Banach algebras, with $\left\{\xi_{i}\right\}_{i \in I}$ a family of isometric involutive antiautomorphisms, $\xi_{i}: A_{i} \rightarrow A_{i}$, such that $\xi_{j} \circ e_{j i}=e_{j i} \circ \xi_{i}$ for $i \leq j$. Write $A=\lim _{\rightarrow} S$; then:

(1) There exists a unique isometric involutive antiautomorphism

$$
\xi: A \rightarrow A
$$

satisfying $\xi \circ e_{i}=e_{i} \circ \xi_{i}$ for any $i \in I$.

(2) If we consider the Banach-Lie subalgebra of $A_{i}^{-}, S k w\left(A_{i}, \xi_{i}\right)$ then

$$
\operatorname{Skw}(S, \xi):=\left(\left\{S k w\left(A_{i}, \xi_{i}\right)\right\}_{i \in I},\left\{\left.e_{j i}\right|_{S k w\left(A_{i}, \xi_{i}\right)}\right\}_{i \leq j}\right)
$$

is a direct system of Banach-Lie algebras and $\lim (\operatorname{Skw}(S, \xi))=$ $S k w\left(\lim _{\rightarrow} S, \xi\right)$.

\section{The Description Theorem}

We proved in [4] that if $L$ is an infinite dimensional simple Lie algebra having a Cartan decomposition (in the sense of $\S 2$ for Banach-Lie algebras with the only difference that $L$ is now written as $L=H \oplus$ $\bigoplus_{\alpha \in \Lambda} L_{\alpha}$ ), relative to a Cartan subalgebra (in the sense of [1]) $H$, and 
such that the nonzero roots spaces are finite dimensional, then $L$ is isomorphic to $\lim S$, where $S=\left(\left\{L_{i}\right\}_{i \in I},\left\{i_{j i}\right\}_{i \leq j}\right)$ is a direct system of simple finite $\overrightarrow{d i m e n s i o n a l ~ L i e ~ s u b a l g e b r a s ~ o f ~ t h e ~ s a m e ~ t y p e ~} A_{l}, B_{l}$, $C_{l}$ or $D_{l}$.

Let us now consider an infinite dimensional topologically simple compact Banach-Lie algebra $L$ having a Cartan decomposition relative to a Cartan subalgebra $H$. By $\S 2, H$ is also a Cartan subalgebra in the sense of [1]. As consequence of Theorem 1 the nonzero root spaces of $L$ satisfy the condition of being finite dimensional. Hence, there is no problem in refining the argument used in the proof of the above result to obtain that $L$ is isometrically isomorphic to $\lim _{\rightarrow} S$, with $S=\left(\left\{L_{i}\right\}_{i \in I},\left\{i_{j i}\right\}_{i \leq j}\right)$ a direct system of simple finite dimensional Banach-Lie subalgebras of the same type $A_{l}, B_{l}, C_{l}$ or $D_{l}$. Finally, the properties of the direct limits given in $\S 3$ let us formulate the following result.

Theorem 4. Suppose that $L$ is an infinite dimensional topologically simple compact Banach-Lie algebra having a Cartan decomposition relative to a Cartan subalgebra $H$. Then $L$ is isometrically isomorphic to some of the following ones:

(1) $A^{-}$, where $A$ is a topologically simple associative Banach algebra.

(2) $\operatorname{Skw}(A, \xi)$ with $A$ as above and $\xi$ an involutive antiautomorphism of $A$.

Acknowledgement. The authors are grateful to Professor Cándido Martín for stimulating discussions during the preparation of this work, and to the referee for his valuable suggestions.

\section{REFERENCES}

[1] Y. Billig and A. Pianzola, On Cartan subalgebras, J. Algebra 171 (1995), 397-412.

[2] F. F. Bonsall and J. Duncan, Complete Normed Algebras. Springer-Verlag, Berlin Heidelberg New York, 1973.

[3] A. J. Calderón and C. Martín, Direct limits of $L^{*}$-triples, Algebra Groups Geom. Palm Harbor, FL. 18 (2001), 223-232.

[4] A. J. Calderón and M. Forero, On infinite dimensional Lie algebras having a Cartan decomposition, Preprint, Universidad de Cádiz (2002).

[5] J. A. Cuenca, A. García and C. Martín, Structure theory for $L^{*}$-algebras, Math. Proc. Camb. Phil. Soc. 107 (1990), 361-365. 
[6] P. De la Harpe, Classical Banach-Lie algebras and Banach-Lie groups of operators in Hilbert spaces, Lecture notes in Math. 285, Springer, Berlin, 1972.

[7] J. R. Schue, Hilbert Space methods in the theory of Lie algebras, Trans. Amer. Math. Soc. 95 (1960), 69-80.

[8] J. R. Schue, Cartan decompositions for $L^{*}$-algebras, Trans. Amer. Math. Soc. 98 (1961), 334-349.

A. J. Calderón Martín, M. Forero Piulestán, Departamento de Matemáticas,

Universidad de Cádiz,

11510 Puerto Real, Cádiz, Spain

ajesus.calderon@uca.es

Received on 8 October 2001 and in revised form on 12 September 2002. 